\title{
Athetoid and Choreiform Hyperkinesias Produced by Caudate Application of Dopamine in Cats
}

\author{
A. R. Coous
}

Department of Pharmacology, University of Nijmegen, The Netherlands

Received December 20, 1971 ; Final Version February 28, 1972

\begin{abstract}
Behavioral changes resembling human athetoid and choreiform hyperkinesias were produced by unilateral injections of L-3,4-dihydroxyphenylalanine (L-DOPA), dopamine, 3-methoxy-tyramine and dexamphetamine in to the rostromedial part of the caput caudati of cats ; saline, noradrenaline and 3,4-dihydroxyphenylacetio acid were ineffective. Pretreatment with the tyrosine hydroxylase inhibitor, $\alpha$-methyl-para-tyrosine, blocked the effects induced by L-DOPA, dopamine and dexamphetamine in a competitive way ; the 3-methory-tyramine effect was blocked in a non-competitive way. Applications of the active compounds into the anteroventral part of the caput caudati were ineffective.

The hypothesis is put forward that normally dopamine-sensitive sites are involved in the manifestation of choreo-athetoid movements. The implications of these findings are considered in view of the efficacy of L-DOPA and $\alpha$-methyl-DOPA. in Parkinson's disease and Huntington's chorea, respectively. In addition, our date are discussed in the light of the recent finding that lesions restricted to the anteroventral part of the caput caudati also result in these hyperkinesias.

Key words: Caudate Nucleus - Chorea-Athetoid - Cats - Dopamine.
\end{abstract}

\section{Introduction}

Since Ehringer and Hornykiewioz reported in 1960 that the con. centration of dopamine in the neostriatum and globus pallidus is reduced in parkinsonism, considerable interest has developed in the neurochemistry of the underlying disorder. At the present time, the introduction of L-3,4-dihydroxyphenylalanine (L-DOPA) appears to be the most important advance in the treatment of Parkinson's disease (Klawans, Ilahi and Shenker, 1970b). Long-term x-DOPA therapy improves the bradykinesia, rigidity, loss of postural reflexes and to a lesser extent the tremor of parkinsonism (Cotzias, Papavasiliou and Gellene, 1969; Yahr, Duvoisin, Schear, Barrett and Hoehn, 1969). In view of the essential role of dopamine in the caudate nucleus (Hornykiewioz, 1966), it is generally accepted that the therapeutic efficacy of L-DOPA is due to the replacement of dopamine in the basal ganglia by exogeneous dopamine derived from L-DOPA (Klawans, 1968).

However, L-DOPA treatment is not without side-effects: in a significant number of patients extremely severe choreo-athetoid movements 
are seen after long-term treatment (Yahr et al., 1969; Papavasiliou, Gellene and Cotzias, 1969). Choreo-athetoid movements are generally imputed to structures such as the subthalamic nucleus, putamen, nucleus accumbens septi and brachium conjunctivum. Paparasiliou et al. (1969), therefore, suggested that the side-effects secondary to long-term treatment of L-DOPA only develop when excesses of dopamine enter neurons from which they are ordinarily excluded; their arguments are in favor of normally dopamine-insensitive sites as the target sites of these side-effects.

On the other hand, in a report describing the behavioral effects of small lesions in cats, Liles and Davis (1969a) provided the information that the caudate nucleus itself is involved in choreo-athetoid hyperkinesias.

In view of these findings the hypothesis may be put forward that the normally dopamine-sensitive sites are also involved as the target sites of the choreo-athetoid movements.

In former experiments it was shown that dopamine, administered unilaterally into the caudate nucleus of cats, induced a stereotyped behavior marked by a decrease of specific units such as licking and cleaning, by a decrease of dynamic units such as standing and walking and by stereotyped movements of the head and the limbs (Cools and van Rossum, 1970; Cools, 1971).

In view of the mentioned considerations the present study was designed to examine in more detail the temporal patterning of the induced limb movements.

\section{Methods}

A detailed account of the experimental technique has already been published (Cools and van Rossum, 1970). Briefly, double barrelled cannulae were stereotaxically implanted into the brain of male cats. Upon complete recovery from the operation, small quantities of drug solutions $(10 \mu l)$ were injected through the injection needle which extended into the brain tissue $2 \mathrm{~mm}$ below the tip of the embedded cannulae. Since it has been shown that dopamine application is ineffective in all caudate areas except from the caput caudati rostromedialis (Cools et al., 1970), brain site co-ordinates for placements discussed in this paper included this area $\left(A_{14-18}, L_{4-6}, H_{14-18}\right)$ and the caput caudati anteroventralis $\left(A_{17-19}, L_{3-5}, H_{13-15}\right)$ which was used as a control area (Fig.1).

The following substances were injected: saline(placebo),L-DOPA-HCl, dopamine-HCl,3-methoxy-tyramine-HCl,noradrenaline-HCl,3,4-dihydroxyphenylacetic acid(DOPAC) and dexamphetamine- $\mathrm{H}_{2} \mathrm{SO}_{4}$; all drugs were dissolved in saline and adjusted to $\mathrm{pH} 4 \mathbf{4}$. In additional experiments pretreatment with the tyrosine hydroxylase inhibitor, $\alpha$-methyl- 


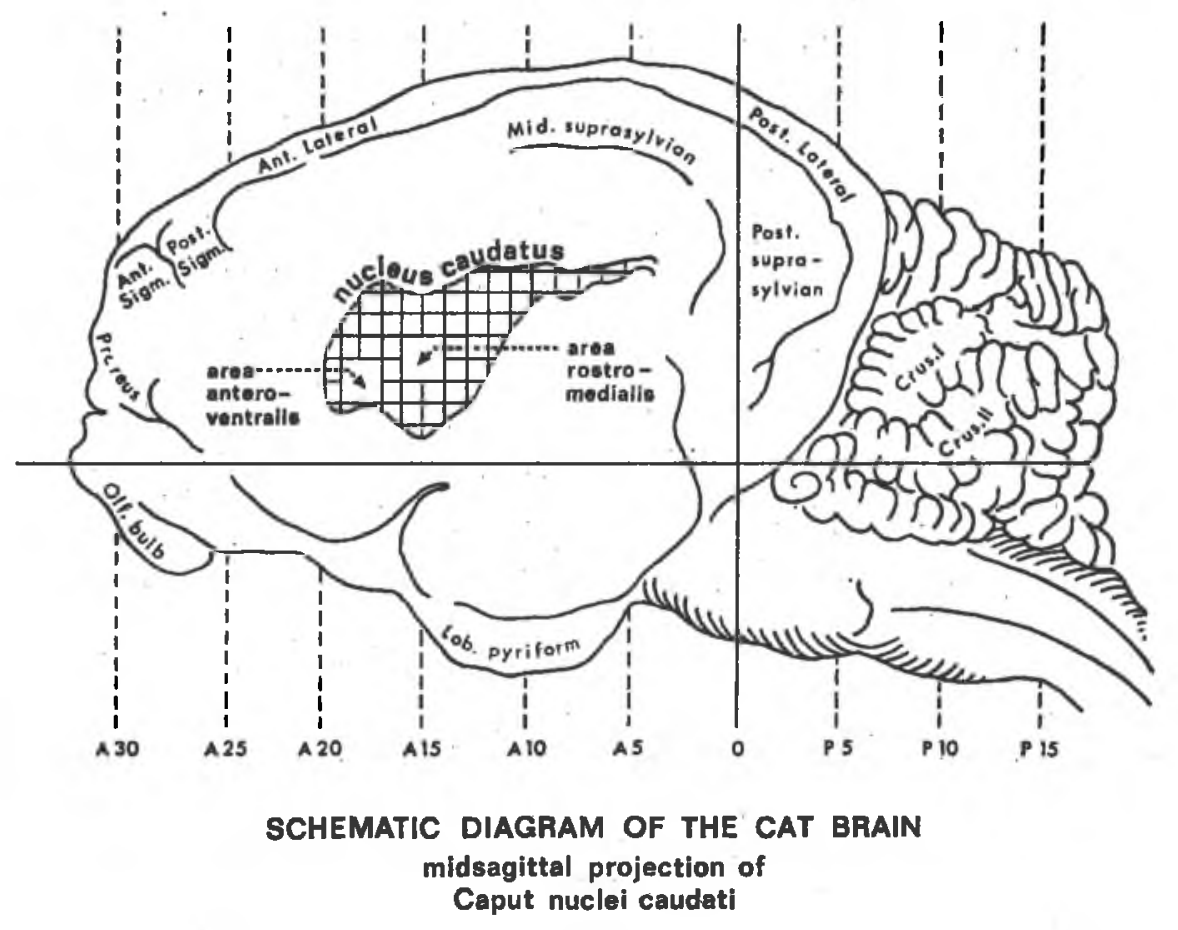

Fig. 1

para-tyrosine ( $\alpha$-MT), was given in order to test the specificity of the induced effects; 20 min prior to the application of dopamine or related compounds $20 \mu \mathrm{g}$ o-MT was injected locally. The temporal patterning of the limbs was recorded during a period of $45 \mathrm{~min}$ divided into nine time test-blocks, $5 \mathrm{~min}$ each: three before and six after the injection. In each six animals (12 injection-loci) were used.

\section{Results}

In general, the abnormal limb movements following an injection of $10 \mu \mathrm{g}$ dopamine into the caput caudati rostromedialis could be divided into two groups:non-patterned movements involving the distal part of the limb and non-patterned movements involving the entire limb.

A. Non-patterned movements involving the distal part of the limb.

When the animal was standing, sitting or lying on its side, these movements began slowly: the limb was lifted from the floor for a couple of seconds and the paw and toes underwent alternating hyperextension and flexion movements. The alternating rhythmic ventriflexions and 
dorsiflexions were slow patterns rather than isolated acts and occurred either at rest or in the course of a voluntary action; in the last case the animal stopped the performance of the voluntary action when the movements appeared. On many occasions the appearance was accompanied by piloerection and curling of the tail. The whole pattern was purposeless, non-adaptive and compulsive in character. It occurred independently of the ongoing activity of the animal and the animal itself had no visual interest in the induced patterning of its limb. The activity was restricted to the forelimb contralateral to the injection site in case of DOPA, dopamine and 3-methoxy-tyramine; it was alternating from one forelimb to the other in case of dexamphetaimne.

B. Non-patterned movements involving the entire limb.

These movements occurred only when the animal was standing or sitting. Usually, they followed up or interrupted the above-mentioned movements. The limb was lifted from the floor and it showed rapid, jerky, shaking-off movements involving the entire limb; these movements were repeated several times in an irregular way. The series of abrupt, gross irregular movements was violent and choreiform in nature. Apart from the fact that the animal had continuous visual interest in these movements, it began to clean and to lick the affected limb in an intense way, when the movements disappeared. These choreiform movements were induced not only in the forelimb contralateral to the injection site, but also in the hindlimb homolateral to the injection site.

In general, both kinds of movements appeared as episodes of which the frequency had a maximum in the middle of the first 5-min period following the injection; at some times the choreiform movements were also present in the second and third 5 -min period. The involuntary and irregular movements of the limbs were always elicited by small doses $(10 \mu \mathrm{g})$ of DOPA, dopamine, 3-methoxy-tyramine and dexamphetamine administered into the caput caudati rostromedialis. No limb movements were noted when saline (placebo), noradrenaline or DOPAC were applied (Table 1$)$; even high doses $(80 \mu \mathrm{g})$ were ineffective. The most effective dose of DOPA and dopamine was $25 \mu \mathrm{g}$ and $80 \mu \mathrm{g}$, respectively; 3-methoxy-tyramine was equally effective in spite of the dose $(10 \mu \mathrm{g}-80 \mu \mathrm{g})$. The effect of dexamphetamine was markedly increased in comparison with the maximum effect of dopamine; not only the intensity of the movements was increased, but also the episode itself was prolonged. However, extending the dose range from $10 \mu \mathrm{g}$ to $144 \mu \mathrm{g}$ yielded no further increase in effect.

Of the brain areas sampled, the movements were. always elicited from the caput caudati rostromedialis, although the intensity and frequency varied considerably between animals. Animals in which the 
Table 1. Quantitative aspects of the athetoid and choreiform episodes in the cat after microinjections into the rostromedial part of the caput caudatia. Numbers of episodes are average rates: $0=$ none; $=1-3 ; 2=4-6 ; 3>7$

\begin{tabular}{|c|c|c|c|c|c|c|}
\hline \multirow{2}{*}{ Compounds } & \multirow{2}{*}{$\frac{\text { dose }}{(\mu \mathrm{g} / 10 \mu \mathrm{l})}$} & \multicolumn{3}{|c|}{ post injeotion } & \multicolumn{2}{|c|}{ time intervals (min) } \\
\hline & & $0-1$ & $2-3$ & $4-6$ & $6-10$ & $11-16$ \\
\hline \multirow[t]{2}{*}{ L-DOPA } & 10.0 & 0 & 1 & 1 & 0 & 0 \\
\hline & 25.0 & $\mathbf{0}$ & 2 & 1 & 1 & $\mathbf{0}$ \\
\hline \multirow[t]{2}{*}{ dopamine } & 10.0 & 0 & 1 & 0 & 0 & o \\
\hline & 80.0 & 0 & 2 & 1 & 1 & 0 \\
\hline \multirow[t]{2}{*}{ DOPAC } & 10.0 & $\mathbf{0}$ & $\mathbf{0}$ & $\mathbf{0}$ & $\mathbf{0}$ & $\mathbf{0}$ \\
\hline & 80.0 & o & 0 & 0 & 0 & 0 \\
\hline \multirow[t]{2}{*}{ 3-methoxytyramine } & 10.0 & o & 1 & 1 & 0 & 0 \\
\hline & 80.0 & 0 & 1 & 1 & 0 & 0 \\
\hline \multirow[t]{2}{*}{ noradrenaline } & 10.0 & 0 & 0 & 0 & 0 & 0 \\
\hline & 80.0 & 0 & 0 & 0 & 0 & 0 \\
\hline \multirow[t]{2}{*}{ dexamphetamine } & 10.0 & 0 & 3 & 2 & 1 & 1 \\
\hline & 144.0 & 0 & 3 & 2 & 1 & 1 \\
\hline saline & 0.09 & 0 & o & 0 & 0 & 0 \\
\hline
\end{tabular}

a The number of limb movements are varying from 1-10 per episode.

compounds were administered into the caput caudati anteroventralis sometimes exhibited these movements; if present, the latency of their appearance was increased from 2-3 $\mathrm{min}$ to $20-24 \mathrm{~min}$ and the frequency was strongly decreased. When $\alpha$-MT was given 20 min prior to the effective compounds, the limb movements induced by low doses $(10 \mu \mathrm{g})$ of DOPA, dopamine, 3-methoxy-tyramine and dexamphetamine were totally suppressed. High doses $(80 \mu \mathrm{g})$ of only DOPA, dopamine and dexamphetamine surmounted the inhibition and induced the originally elicited involuntary and irregular patterns; in contrast, 3-methoxy-tyramine remained ineffective in spite of high doses $(80 \mu \mathrm{g})$.

\section{Discussion}

The non-patterned movements involving the distal part of the limb and the non-patterned movements involving the entire limb have to be determined as athetoid and choreiform movements respectively in reference to the terminology used by Liles and Davis in their lesion study (1969a). In fact, no distinguishable differences could be found between our observations and their detailed descriptions. However, small differences were noted with respect to the side of occurence: in the lesion study the athetoid movements normally appeared in both forelimbs, while they occasionally occurred in the forelimb contralateral to the lesion site; in our study these movements were always restricted to the forelimb contralateral to the injection site in the case of DOPA, 
dopamine and 3-methoxy-tyramine, while they were alternating from one forelimb to the other in the case of dexamphetamine.

Although 3-methoxy-tyramine appeared to be just as effective as dopamine, the non-competitive inhibition of its effect by the dopaminesynthesis inhibitor, $\alpha$-MT, and the ineffectiveness of the other dopamine metabolites, DOPAC and noradrenaline, indicate that the abnormal patternings of the limb movements have to be regarded as "dopaminespecific" effects; the effectiveness of high doses of only DOPA, dopamine and dexamphetamine following inhibition of the dopamine-synthesis strengthens this assumption.

The observation that dopamine and related compounds were only effective in the caput caudati rostromedialis is in agreement with our previously reported finding that the dopamine-sensitive sites are restricted to the rostromedial area (Cools and van Rossum, 1970).

In regard to the data of Liles and Davis (1969a) the conclusion can be drawn that two clearly distinct areas of the caudate nucleus are involved in these symptoms: the rostromedial area following dopamine application and the anteroventral area following electrolytic lesion. Since neither dopamine application into the anteroventral area nor lesion of the rostromedial area did lead to the mentioned hyperkinesias, these areas must have different functions. It has been shown that the head of the caudate nucleus represents localized functional regions: the inhibiting anteroventral region and the facilitating rostromedial region ${ }^{1}$ (Liles and Davis, $1969 \mathrm{~b}$ ). In a previous report we have found evidence that dopamine in the rostromedial area might induce activation of inhibiting neurons (Cools, 1971). In view of these findings it is reasonable to suggest that the dopamine-sensitive sites which are restricted to the caput caudati rostromedialis belong to very small inhibiting neurons which are projecting to the caput caudati anteroventralis; for activation of these interneurons or lesion of the cell bodies controlled by these neurons results in the same effect. Although the nature of these interneurons is so far unknown, extremely small caliber neurons have been observed previously in the head of the caput caudati (Adinolfi, 1967). Experiments in order to test this are in progress. The most important result, however, is the discovery that the choreoathetoid hyperkinesias are elicited from the caudate nucleus in which dopamine normally occurs in terminal structures of nerve-endings (Fuxe, 1965). Thus, activation or overactivation of normally dopamine-sensitive sites leads to choreo-athetoid hyperkinesias.

In view of these data, the hypothesis can be put forward that normally dopamine-sensitive sites (of the caudate nucleus) are also involved

1 Rostromedial area correponds partially to the posterodorsal area of Liles and Davis (1969a and b) 
as the target sites of choreo-athetoid movements observed in human beings. This working hypothesis is formulated on the basis of three adequately proven facts: a) choreo-athetoid movements are induced in a significant number of parkinsonian patients after long-term L-DOPA treatment (see Introduction); b) L-DOPA orally administered in animals induces a highly specifio accumulation of dopamine in the caudate nucleus (Pletscher, 1969); c) L-DOPA or dopamine locally applied into the caudate nucleus of cats induces movement disorders which are strikingly similar to the behavior neurologists have seen in patients treated with L-DOPA and in patients with Huntington's chorea.

This may have the implication that the enthusiasm of L-DOPA treatment has to be tempered: there is no evidence that the intracaudate dopamine-level involved in the therapeutic effects is different from the intracaudate dopamine-level involved in the side-effects: as mentioned, on reaching therapeutic L-DOPA-levels the choreo-athetoid hyperkinesias appear in a large number of parkinsonians (Papavasiliou et al., 1969).

On the other hand, the continuing controversy of L-DOPA efficacy may also be explained on the basis of the striatal oholine-dopamine imbalance, the importance of which has been stressed from results of several animal studies (Connor, 1966; Arnfred and Randrup, 1967; Scheel-Krüger, 1970). Although it is generally accepted that the imbalance in the normal relationship of dopamine and acetylcholine in parkinsonians results from the primary involvement of a deficient dopamine-input in the striatum (Hornykiewicz, 1966; Klawans, 1968), the antiparkinson activity of anticholinergic drugs (Feldberg, 1945; Klawans, 1968), the parkinson-reinforcing activity of cholinesterase inhibitors (Duvoisin, 1967) and the ineffectiveness of L-DOPA in some parkinsonians (Klawans, Ilahi and Shenker, 1970 b) do not exclude that the imbalance in some patients may result from a primary involvement of the cholinergic input: in other words, an imbalance resulting from an overactivity of the striatal choline system rather than from a deficiency of the striatal dopamine system. It is reasonable to suggest that I-DOPA treatment of these patients always results in an overactivation of the dopamine-sensitive sites and, thus, in the chorea-athetoid hyperkinesias. However, it remains to be investigatcd whether this occurs in the patients which are not alleviated by L-DOPA. Summarizing, these considerations indicate that further experience will be required before L-DOPA can be accepted as the most successful drug in the treatment of Parkinson's disease. As a final remark, the described movements induced by locally applied L-DOPA or dopamine support the hypothesis that choreiform movements observed in Huntington's chorea are due to an overactivation of the dopamine-sensitive sites in the caudate nucleus (Klawans, 1970 a). According to this hypothesis inhibition of the overactivation 
must consequently lead to the suppression of these movements; this indicates that dopamine-inhibitors may have some therapeutic value in Huntington's chorea.

Indeed, Papavasiliou et al. (1969) reported that $\alpha$-methyl-DOPA, a depletor of catecholamines, induced a significant diminution of these movements. On the other hand, cholinesterase inhibitors also induce a reduction of the choreiform movements (Aquilonius and Sjöstrom, 1971). This again suggests that motor disorders of the basal ganglia result from the difference in the absolute activities of dopamine and acetylcholine rather than from the absolute activity of only one of them.

\section{Bibliography}

Adinolf, A.: Obervations on the fine structure of the feline caudate nucleus. Anat. Reo. 157, 203 (1967).

Aquilonius, S. M., Sjöstrom, R.: Cholinergic and dopaminergio mechaniscs in Huntington's chorea. Life Sci. 10, pt. I, 405-414 (1917).

Arnfred, T., Randrup, A.: Cholinergio mechanism in brain inhibiting amphetamineinduced atereotyped behavior. Acta pharmacol. (Kbh.) 26, 384-394 (1968).

Connor, J. D., Rossi, G. V., Raker, W. W.: Analysis of tremor induced by injection of cholinergic agents into the caudate nucleus. Int. J. Neuropharmacol. 5, $207-216$ (1966).

Cools, A. R.: The function of dopamine and its antagonism in the caudate nuclous of cats in relation to the stereotyped behaviour. Arch. int. Pharmacodyn. 194, 259-269 (1971).

- van Rossum, J. M.: Caudate dopamine and stereotyped behaviour of cats. Arch. int. Pharmacodyn. 187, 163-173 (1970)

Cotzies, G. C., Papavasiliou, P. S., Gellene, R.: Modification of parkinsonism: Chronic treatment with L-dope. New Engl. J. Med. 280, 337-345 (1969).

Duvoisin, R. C.: Cholinergio-anticholinergic antagonism in parkinsonism. Arch. Neurol. (Chic.) 17, 124-136 (1967).

Ehringer, H., Hornykiewicz, O.: Verteilung von Noradrenalin und Dopamin (3-Hydroxytyramin) im Gehirn des Mensehen und ihr Verhalten bei Erkrankungen des extrapyramidalen Systems. Klin. Wschr. 88, 1236-1239 (1960).

Feldberg, W.: Present views on the mode of action of acetylcholine in the central nervous system. Physiol. Rev. 25, 596 (1945).

Fure, K.: Evidence for the existence of monosmine neurons in the central nervous system. IV. Distribution of monoamine nerve terminals in the central nervous system. Acta physiol. soand. 64 (Suppl.), 37-84 (1965).

Hornykiewicz, O.: Dopamine (3-Hydroxytyramine) and brain function. Pharmacol. Rev. 18, 925-964 (1966).

Klawang, H. L. : The pharmacology of parkinsonism. Die. nerv. Syst. 29, 805-816 (1868).

- A pharmacologic Analysis of Huntington's Chorea. Europ. Neurol. 4, 148-163 (1970a).

- Ilahi, M. M., Shenker, D.: Theoretical Implications of the use of L-Dopa in Parkinsonism. Acta neurol. scand. 46, 409-441 (1970 b).

Liles, S. L., Davis, G. D.: Athetoid and ohoreiform hyperkinesias produced by caudate lesions in the oat. Soience 164, 195-197 (1969a).

- - Electrocortical effects of caudate stimulations whioh alter cortically induced movement. J. Neurophysiol. 82, 574-582 (1969b). 
Papavasiliou, P.S., Gellene, R.; Cotzias, G. C.: Modification of parkinsonism: Dyskinesias accompanying treatment with dopa. In: Paychotropic drugs and dysfunctions of the basal ganglia, G. E. Crane and R. Gardner, eds., pp. 140 to 143. Public Health Service Publication no. 1938 (1969).

Pletscher, A.: Pharmacological changes of the dopamine metabolism in the basal ganglia. In: Psychotropic drugs and dysfunctions of the basal ganglia. G. E. Grane, R. Gardner, eds., 122-130. Public Health Service Publication no. 1938 (1969).

Scheel-Krüger, J.: Central effects of anticholinergic druge measured by the apomorphine test in mice. Acta pharmacol. (Kbh.) 98, 1-16 (1970).

Yahr, M. D., Duvoisin, R. C., Schear, M. J., Barrett, R. E., Hoehn, M. M.: Treatment of parkinsonism with Levodopa. Arch. Neurol. (Chic.) 21, 343-354 (1969).

Dr. A. R. Cools

Department of Pharmacology

University of Nijmegen

Nijmegen, The Netherlands 